\title{
ON A RESULT OF HADAMARD CONCERNING THE SIGN OF THE PRECESSION OF A HEAVY SYMMETRICAL TOP
}

\author{
J. B. DIAZ AND F. T. METCALF
}

Consider Lagrange's integrable case of the motion of a rigid body about a fixed point, when the center of gravity of the body lies on the polar axis of the spheroid of inertia of the body [1, case II, pp. 216249].

In $[1, \S 110$, pp. $233-235]$ one finds "An interesting proof that the precession for a complete period has the same sign as $\omega_{k}$ (which) has been given by Hadamard" (see [2]). Hadamard's proof employs the theory of residues of functions of a complex variable. Quite recently (see [3], which cites the recent literature), upper and lower bounds for the apsidal angle in the theory of the heavy symmetrical top have been obtained by an elementary method, not relying upon the theory of residues. This same direct method applies equally well in deducing Hadamard's result. This application will be carried out here, in the notation of [1], for the immediate comparison of the two methods.

The argument requires the preliminary evaluation of two simple definite integrals:

$$
\begin{aligned}
\pi & =\int_{u_{3}}^{u_{2}} \frac{\left(\left(1+u_{2}\right)\left(1+u_{3}\right)\right)^{1 / 2} d u}{(1+u)\left(\left(u_{2}-u\right)\left(u-u_{3}\right)\right)^{1 / 2}} \\
& =\int_{u_{3}}^{u_{2}} \frac{\left(\left(1-u_{2}\right)\left(1-u_{3}\right)\right)^{1 / 2} d u}{(1-u)\left(\left(u_{2}-u\right)\left(u-u_{3}\right)\right)^{1 / 2}}, \quad-1<u_{3}<u_{2}<1 .
\end{aligned}
$$

Just put $u=-v$ in the second integral to obtain the first; and put $1+u=\left(1+u_{3}\right)\left(1+u_{2}\right)\left(1+v^{2}\right)\left[\left(1+u_{2}\right)+\left(1+u_{3}\right) v^{2}\right]^{-1}, 0 \leqq v<\infty$, in the first.

The problem $[1$, p. 233$]$ is to show that the definite integral

$$
\begin{aligned}
& \frac{a^{1 / 2}}{b \omega_{k}}\left(\psi-\psi_{0}\right)=\int_{u_{3}}^{u_{2}} \frac{u_{4}-u}{1-u^{2}} \frac{d u}{\left(\left(u_{1}-u\right)\left(u_{2}-u\right)\left(u-u_{3}\right)\right)^{1 / 2}}, \\
& -1<u_{3}<u_{4}<u_{2}<1<u_{1} \text {, }
\end{aligned}
$$

is positive, the square root being positive. The case under consideration, where $u_{4}$ satisfies $u_{3}<u_{4}<u_{2}$, is that in which "loops" occur [ 1 , Figure 61, p. 242], and is the only "doubtful" case; if $u_{4} \geqq u_{2}$ or $u_{4} \leqq u_{3}$ then the state of affairs is evident.

Received by the editors March 8, 1962. 
Now, since

$$
\frac{u_{4}-u}{1-u^{2}}=\frac{1}{2}\left[\frac{1+u_{4}}{1+u}-\frac{1-u_{4}}{1-u}\right],
$$

it follows that

$$
\begin{aligned}
2 \int_{u_{3}}^{u_{2}} \frac{u_{4}-u}{1-u^{2}} \frac{d u}{\left(\left(u_{1}-u\right)\left(u_{2}-u\right)\left(u-u_{3}\right)\right)^{1 / 2}} \\
\quad=\int_{u_{3}}^{u_{2}} \frac{1+u_{4}}{1+u} \frac{d u}{\left(\left(u_{1}-u\right)\left(u_{2}-u\right)\left(u-u_{3}\right)\right)^{1 / 2}} \\
-\int_{u_{3}}^{u_{2}} \frac{1-u_{4}}{1-u} \frac{d u}{\left(\left(u_{1}-u\right)\left(u_{2}-u\right)\left(u-u_{3}\right)\right)^{1 / 2}} .
\end{aligned}
$$

However,

$$
\frac{1}{\left(u_{1}+1\right)^{1 / 2}}<\frac{1}{\left(u_{1}-u\right)^{1 / 2}}<\frac{1}{\left(u_{1}-1\right)^{1 / 2}}, \quad-1<u_{3} \leqq u \leqq u_{2}<1<u_{1} .
$$

Hence

$$
\begin{aligned}
& 2 \frac{a^{1 / 2}}{b \omega_{k}}\left(\psi-\psi_{0}\right) \\
& \quad>\pi\left[\frac{1+u_{4}}{\left(\left(u_{1}+1\right)\left(1+u_{2}\right)\left(1+u_{3}\right)\right)^{1 / 2}}-\frac{1-u_{4}}{\left(\left(u_{1}-1\right)\left(1-u_{2}\right)\left(1-u_{3}\right)\right)^{1 / 2}}\right] \\
& \quad=0
\end{aligned}
$$

since, from $[1$, p. 221$]$, when $u_{3}<u_{4}<u_{2}$,

$$
\frac{1-u_{4}}{1+u_{4}}=\left(\frac{\left(u_{1}-1\right)\left(1-u_{2}\right)\left(1-u_{3}\right)}{\left(u_{1}+1\right)\left(u_{2}+1\right)\left(u_{3}+1\right)}\right)^{1 / 2} \text {. }
$$

\section{REFERENCES}

1. W. D. MacMillan, Dynamics of rigid bodies, Dover, New York, 1960.

2. J. Hadamard, Sur la précession dans le mouvement d'un corps pesant de revolution fixe par un point de son axe, Bull. Sci. Math. 19 (1895), 228-230.

3. J. B. Diaz and F. T. Metcalf, Upper and lower bounds for the apsidal angle in the heory of the heavy symmetrical top (submitted to the Journal of Mathematical Physics).

Institute for Fluid Dynamics and Applied Mathematics, University of MARYLAND AND

U. S. Naval Ordnance Laboratory. 